\title{
What is the impact of local control in Ewing sarcoma: analysis of the first Brazilian collaborative study group - EWING1
}

Ricardo G. Becker ${ }^{1 *}$, Lauro J. Gregianin ${ }^{2,3}$, Carlos R. Galia ${ }^{4}$, Reynaldo Jesus-Garcia Filho ${ }^{5}$, Eduardo A. Toller ${ }^{6}$, Gerardo Badell7, Suely A. Nakagawa ${ }^{8}$, Alexandre David ${ }^{9}$, André M. Baptista ${ }^{10}$, Eduardo S. Yonamime ${ }^{11}$, Osvaldo A. Serafini ${ }^{12}$, Valter Penna ${ }^{13}$, Julie Francine C. Santos ${ }^{14}$, Algemir L. Brunetto ${ }^{15}$ and On behalf of the Brazilian Collaborative Study Group of Ewing Family of Tumors - EWING1 and the Brazilian Society of Pediatric Oncology - SOBOPE

\begin{abstract}
Background: Relapse in localized Ewing sarcoma patients has been a matter of concern regarding poor prognosis. Therefore, we investigated the impact of local control modality (surgery, surgery plus radiotherapy, and radiotherapy) on clinical outcomes such as survival and recurrence in patients with non-metastatic Ewing sarcoma treated on the first Brazilian Collaborative Group Trial of the Ewing Family of Tumors (EWING1).

Methods: Seventy-three patients with localized Ewing sarcoma of bone aged $<30$ years were included. The treating physicians defined the modality of local control based on the recommendations of the coordinating center and the patient and tumor characteristics. Possible associations of local control modality with local failure (LF), disease-free survival (DFS), event-free survival (EFS), overall survival (OS), and clinical characteristics were analyzed.

Results: Mean patient age was 12.8 years (range, 2 to 25 years) and median follow-up time was 4.5 years (range, 2. 3 to 6.7 years). Forty-seven patients underwent surgery, 13 received radiotherapy, and 13 received both. The 5-year EFS, OS, and DFS for all patients was $62.1 \%, 63.3 \%$, and $73.1 \%$, respectively. The 5 -year cumulative incidence (CI) of LF was $7.6 \%$ for surgery, $11.1 \%$ for radiotherapy, and $0 \%$ for postoperative radiotherapy (PORT) $(p=0.61)$. The 5 -year EFS was $71.7 \%$ for surgery, $30.8 \%$ for radiotherapy, and $64.1 \%$ for PORT ( $p=0.009$ ).

Conclusions: There was a significant effect of local control modality on EFS and OS in the study. Surgery and PORT modalities yielded very close results. The group treated with radiotherapy alone had considerably worse outcomes. This may be confounded by greater risk factors in these patients. There was no significant effect of local control modality on the CI of LF and DFS.
\end{abstract}

Keywords: Ewing sarcoma, Local control, Radiation oncology, Surgery, Bone tumors, Orthopedics

\footnotetext{
* Correspondence: rbecker@hcpa.edu.br; formato2@scientific.com.br

${ }^{1}$ Service of Orthopedics and Traumatology, Hospital de Clínicas de Porto

Alegre (HCPA), Rua Ramiro Barcelos, 2350, Bairro Santa Cecilia, Porto Alegre,

RS, 90035-903, Brazil

Full list of author information is available at the end of the article
} 


\section{Background}

Ewing sarcoma (ES) is a small round cell malignancy of bone and soft tissue that usually occurs in individuals aged 5 to 20 years. Five-year overall survival (OS) for patients with localized disease ranges from 65 to $75 \%$, while disease relapse after local control reduces survival to less than 25\% [1-8]. Multicenter trials have demonstrated the importance of aggressive chemotherapy treatment and local control of the primary tumor. Successful local control rates have improved to $74-93 \%$ with the introduction of a multidisciplinary and collaborative approach [9-12].

Current ES treatment includes induction chemotherapy, local control of the primary tumor, and consolidation chemotherapy. Surgery alone or in combination with radiation has traditionally been considered a good choice for resectable ES, while most unresectable tumors have been treated with radiation alone. However, recent studies have reported worse local recurrence and survival rates in patients treated with radiotherapy alone compared to surgery and postoperative radiotherapy (PORT). These findings have been associated with risk factors that are present in irradiated patients [12-19].

For the first time in Brazil, data on local control of ES were analyzed within a single multicenter protocol. We used a cohort of patients with localized ES treated on the EWING1 trial (first Brazilian Collaborative Group Trial for treatment of Ewing sarcoma family of tumors [ESFT]) [20] to evaluate different local control strategies and their association with risk factors, relapse, and survival.

\section{Methods}

\section{Patient enrollment}

The study was approved by the institutional review board of Hospital de Clínicas de Porto Alegre through the Office of Research and Graduate Studies (IRB No. 00000921). All patients signed an informed consent form prior to their inclusion in the EWING1 trial from 2003 to 2010 (original trial, IRB No. 03363, date: October 15, 2003).

Patients with localized ES of bone treated between 2003 and 2010 according to the EWING1 trial were eligible for the study. Patients were allocated to low-risk (LRG) or high-risk (HRG) groups, where high-risk patients were defined as those with unresectable tumors, tumors of the pelvis, and lactate dehydrogenase (LDH) levels $\geq 1.5$ times the upper limit of normal ( $x$ ULN). Tumor size was assessed on magnetic resonance imaging (MRI) and computed tomography (CT) scans before starting induction chemotherapy and categorized into $\leq 8 \mathrm{~cm}$ (small tumors) and $>8 \mathrm{~cm}$ (large tumors). Chemotherapy response was defined as good or poor according to the necrosis index (>95\% or $\leq 95 \%$, respectively) $[21,22]$.

Patients were treated at 15 centers located in 6 states in Brazil, and one in Uruguay. Each center's institutional review board approved the treatment protocols, and written informed consent was obtained for all patients at enrollment.

\section{Treatment}

In the EWING1 trial, the induction chemotherapy consisted of two courses of ifosfamide/carboplatin/etoposide (ICE) and two courses of vincristine/doxorubicin/cyclophosphamide (VDC), followed by local control. After local treatment, LRG patients received 10 additional alternating cycles of ifosfamide/etoposide (IE) with VDC, while HRG patients received two additional cycles of ICE at the end of the consolidation therapy. Details of the treatment plan and timing of local control have been published previously [20].

Local control modality was defined based on the experience of treating physicians within each participating institution; however, the coordinating center established some criteria based on the patient and tumor characteristics to standardize the choice of local control. Patients with tumors that were amenable to resection with adequate margins, regardless of size, response to chemotherapy, or location, should be treated surgically. Cases with positive surgical margins, in which wide resection was not possible due to high morbidity, should receive PORT. The dose of PORT was defined as 45 Gy for marginal resections and 55.8 Gy for intralesional resections. The presence of necrotic tissue, even in the absence of viable ES cells, was considered incomplete resection and treated with $55.8 \mathrm{~Gy}$. Patients with tumors of the ribs, with a pleural effusion contiguous to a primary lesion, should also receive PORT.

Definitive radiation was given to patients when wide resection could cause high morbidity or mutilation, and in unresectable tumors. Radiation was planned according to the X-ray, CT, and MRI when available. Radiotherapy was delivered to the original tumor volume with a 2-cm margin and a total dose of $55.8 \mathrm{~Gy}$ at $1.8 \mathrm{~Gy} /$ fraction started during week 11. At the end of treatment, it was established that patients would be followed up every 3 months during the first 2 years, then every 6 months for 5 years, and annually thereafter.

Recurrence was classified as local or systemic. For analysis purposes, any local recurrence was defined as local failure (LF) and systemic recurrence as distant failure (DF). Combined recurrences were included in the systemic group. The classification of the local control modality received by each patient was determined according to all interventions performed at the local tumor site up to and including the start of consolidation therapy. Local control was classified into one of three procedures: surgery, radiotherapy, or surgery plus radiotherapy. Overall survival (OS), event-free survival (EFS), and diseasefree survival (DFS) were defined as the time from the end of all local control measures until a respective event 
occurs or last patient contact, at which time the patient was censored. Patients who experienced disease progression, second malignant neoplasm, or death were scored as having experienced an event.

\section{Statistics}

The outcome measures were OS, EFS, DFS, and cumulative incidence (CI) of LF and DF timed from the completion of local control therapy, as calculated by the Kaplan-Meier method. The CI of each type of event was calculated for each method of local control and compared by the log-rank test. Associations between categorical variables were analyzed using Pearson's chi-square test. The Mann-Whitney test was used to compare medians for radiation dose. The association between local control modality and event risk was analyzed using univariate and multivariate Cox proportional-hazards regression models. The hazard ratio (HR) and 95\% confidence interval $(95 \% \mathrm{CI})$ were used as the measure of effect.

\section{Results}

\section{Patients selection and characteristics}

Data from 73 patients (45 males and 28 females, mean age of 12.8 years) with localized bone disease submitted to local control were selected from a total of 175 patients (96 with localized bone and extraosseous ES and 79 with metastatic bone and extraosseous ES) of the EWING1 trial. The median follow-up time of patients in this study was 4.5 years (range, 2.3 to 6.7 years). Forty-three tumors (58.9\%) were located in the extremities, $10(13.7 \%)$ in the pelvis, $10(13.7 \%)$ in the chest wall, $6(8.2 \%)$ in the spine, and $4(5.5 \%)$ in other sites $(p>0.001)$. Thirty-eight (52.1\%) patients were allocated as LRG and 35 (47.9\%) patients as HRG $(p<0.001)$. Pelvic tumors were relatively more likely to receive radiotherapy than surgery alone. On the other hand, non-pelvic tumors were more frequently treated with surgery $(p=0.012)$. Tumor size $\leq 8 \mathrm{~cm}$ vs $>8 \mathrm{~cm}$ was not significantly associated with the local control modality performed $(p=0.12)$. The response to chemotherapy was poor (necrosis index $\leq 95 \%$ ) in $56 \%$ and good (>95\%) in $44 \%$ of patients. Of 68 patients with complete LDH records, only 15 (22\%) had LDH $\geq 1.5 \mathrm{x}$ ULN and were more likely to have a surgical procedure $(66.6 \%)$ than radiotherapy alone $(33.3 \%)(p=0.05)$. The median radiation dose was 50.4 Gy for both groups (range, 45.0 to $55.9 \mathrm{~Gy})$.

Of 43 patients with tumors of the extremities, almost all underwent surgical treatment $(n=41,95.4 \%)$, while only $2(4.6 \%)$ received radiotherapy alone. Of 16 patients with tumors of the pelvis and spine, only 6 (37.5\%) underwent surgery, while $10(62.5 \%)$ received radiotherapy alone $(p<0.001)$ (Table 1$)$.

\section{Overall analysis}

The estimated 5-year EFS, OS, and DFS for all 73 patients was $62.1 \%, 63.3 \%$, and $73.1 \%$, respectively. The 5 -year CI of LF and DF was $6.9 \%$ and $14.7 \%$, respectively. Sixty-eight patients had complete information on local or distant recurrence. Only 4 had isolated LF, and 11 had DF combined or not with LF (Table 2; Figs. 1, 2, and 3).

The 5-year EFS was not statistically different according to tumor size $\leq 8 \mathrm{~cm}$ vs $>8 \mathrm{~cm}$ at presentation $(61.1 \%$ vs $58.1 \%, \mathrm{HR}=1.07 ; P=0.89)$, pelvic location $(41.1 \%$ vs $66.7 \%, \mathrm{HR}=1.47 ; p=0.44), \mathrm{LDH}$ levels $<1.5$ vs $\geq 1.5 \mathrm{x}$ ULN (63.1\% vs $51.3 \%, \mathrm{HR}=1.11 ; p=0.83)$, or radiation dose $(\mathrm{HR}=0.99 ; p=0.56)$. LRG and HRG patients had EFS rates of $73.7 \%$ and $48.2 \%$ and LF rates of $5.6 \%$ and $8.3 \%$, respectively $(p=0.16)$ (Table 2 ).

On multivariate analysis, definitive radiotherapy, age $>15$ years and HRG were not associated with a higher risk of any event (Table 3 ).

\section{Local control analysis}

The 5-year EFS was $30.8 \%$ for patients submitted to definitive radiotherapy (13 patients), $64.1 \%$ for surgery plus radiotherapy (13 patients), and $71.7 \%$ for surgery alone (47 patients) $(p=0.009)$. There was no significant difference in LF rates by local control modality $(p=0.61)$, and the LF rates were the same at 2 and 5 years of follow-up: $7.6 \%$ in the surgery group, $11 \%$ in the radiotherapy group, and $0 \%$ in the PORT group $(p=0.62)$. Considering all 15 patients with local or systemic recurrence, the CI of LF and DF at both 2 and 5 years was $11 \%$ for radiotherapy alone, $16.7 \%$ for surgery plus radiotherapy, and $25 \%$ for surgery alone $(p=0.64)$. The local disease control rate was $78 \%$.

\section{Discussion}

Small round cells tumors such as ES are usually good responders to irradiation. Consequently, radiotherapy has been an important option for local control either alone or with surgery. However, radiotherapy is not free from complications at the primary tumor sites. Soft tissue fibrosis, osteonecrosis, impaired long-bone growth, secondary malignancies, and up to $35 \%$ rate of local recurrence have been related to high-dose irradiation [5, 9-13, 23].

On the other hand, development of orthopedic endoprostheses has enabled surgeons to perform non-mutilating procedures with adequate margins in ES patients. Continuous advances have introduced structural auto and allografts in surgical reconstructions, thus offering more biological treatment options. Therefore, amputation has become extremely infrequent in ES [24, 25].

The presence of marginal or contaminated margins is still the main indication for PORT in the treatment of ES. Conversely, PORT has been routinely used in patients with poor response to chemotherapy as well as in large- 
Table 1 Characteristics of the Sample according to the Local Control Modality

\begin{tabular}{|c|c|c|c|c|c|}
\hline \multirow[t]{3}{*}{ Variables } & \multirow{3}{*}{$\begin{array}{l}\text { Total sample } \\
\text { n (\%) }\end{array}$} & \multicolumn{3}{|c|}{ Local Control Modality } & \multirow[t]{3}{*}{$p$} \\
\hline & & Surgery & Surgery + Radiotherapy & Radiotherapy & \\
\hline & & $n(\%)$ & n (\%) & n (\%) & \\
\hline All patients & $73(100)$ & $47(64.4)$ & $13(17.8)$ & $13(17.8)$ & \\
\hline Age group & & & & & 0.753 \\
\hline$\leq 15$ years & $51(69.9)$ & $34(72.3)$ & $8(61.5)$ & $9(69.2)$ & \\
\hline$>15$ years & $22(30.1)$ & $13(27.7)$ & $5(38.5)$ & $4(30.8)$ & \\
\hline Sex & & & & & 0.035 \\
\hline Male & $45(61.6)$ & $33(70.2)^{a}$ & $4(30.8)$ & $8(61.5)$ & \\
\hline Female & $28(38.4)$ & $14(29.8)$ & $9(69.2)^{a}$ & $5(38.5)$ & \\
\hline Risk group & & & & & $<0.001$ \\
\hline Low & $38(52.1)$ & $33(70.2)^{a}$ & $5(38.5)$ & $0(0.0)$ & \\
\hline High & $35(47.9)$ & $14(29.8)$ & $8(61.5)$ & $13(100)^{a}$ & \\
\hline Tumor size & & & & & 0.124 \\
\hline$\leq 8 \mathrm{~cm}$ & $18 / 45(40.0)$ & 10/31 (32.3) & 4/9 (44.4) & 4/5 (80.0) & \\
\hline$>8 \mathrm{~cm}$ & $27 / 45(60.0)$ & 21/31 (67.7) & $5 / 9(55.6)$ & $1 / 5(20.0)$ & \\
\hline Necrosis Index & & & & & 1.000 \\
\hline$\leq 95 \%$ & $28 / 50(56.0)$ & 23/42 (54.8) & $5 / 8(62.5)$ & NA & \\
\hline$>95 \%$ & $22 / 50(44.0)$ & 19/42 (45.2) & $3 / 8(37.5)$ & NA & \\
\hline LDH & & & & & 0.052 \\
\hline$\geq 1.5 \times$ ULN & 15/68 (22.1) & 6/45 (13.3) & $4 / 10(40.0)$ & $5 / 13(38.5)$ & \\
\hline$<1.5 \times$ ULN & 53/68 (77.9) & $39 / 45(86.7)$ & $6 / 10(60.0)$ & $8 / 13(61.5)$ & \\
\hline Recurrence & & & & & 0.509 \\
\hline No & 53/68 (77.9) & $34 / 46(73.9)$ & 10/12 (83.3) & 9/10 (90.0) & \\
\hline Local & 4/68 (5.9) & $3 / 46(6.5)$ & 0/12 (0.0) & $1 / 10(10.0)$ & \\
\hline Systemic & $11 / 68(16.2)$ & 9/46 (19.6) & $2 / 12(16.7)$ & 0/10 (0.0) & \\
\hline Site location & & & & & $<0.001$ \\
\hline Spine & $6(8.2)$ & $0(0.0)$ & $1(7.7)$ & $5(38.5)^{a}$ & \\
\hline Chest wall & $10(13.7)$ & $6(12.8)$ & $4(30.8)^{a}$ & $0(0.0)$ & \\
\hline Pelvis & $10(13.7)$ & $3(6.4)$ & $2(15.4)$ & $5(38.5)^{a}$ & \\
\hline Proximal extremity & $21(28.8)$ & $17(36.2)^{a}$ & $2(15.4)$ & $2(15.4)$ & \\
\hline Distal extremity & $22(30.1)$ & $19(40.4)^{\mathrm{a}}$ & $3(23.1)$ & $0(0.0)$ & \\
\hline Other & $4(5.5)$ & $2(4.3)$ & $1(7.7)$ & $1(7.7)$ & \\
\hline Radiation dose $(G y)^{b}$ & $50.4(45-55.8)$ & NA & $50.4(45-54.9)$ & $50.4(45-55.8)$ & 0.801 \\
\hline
\end{tabular}

${ }^{a}$ Statistically significant association by adjusted residual analysis at $5 \%$ significance level

${ }^{\mathrm{b}}$ Expressed as median (25th-75th percentile)

$L D H$ lactate dehydrogenase, $N A$ not applicable, ULN upper limit of normal

volume tumors in European centers. The current consensus on the type of local treatment of ES follows criteria based on the patient and tumor characteristics and, not less important, on the level of experience of treating physicians [12-19].

The heterogeneity of clinical factors may be a source of confusion when following the guidelines for local treatment in ES $[6,26]$. Yock et al. evaluated the impact of the local control modality for localized ES in a non-randomized study including 75 patients with pelvic bone disease. There was no difference in recurrence rates or survival between the different local control methods. However, patients with larger tumors were more likely to receive combined surgery plus radiotherapy $(p=0.013)$ [19]. Similarly, in the EWING1 trial, there was no difference in recurrence rate (LF) between the different treatment modalities, and larger tumors were more likely to receive surgery and PORT than radiotherapy $(p=0.12)$. Nevertheless, we believe that the limited size of the sample and the inability to control for confounding factors may be reflected in the results. 
Table 2 Results of univariate analysis for possible independent variables associated with death and EFS

\begin{tabular}{|c|c|c|c|c|c|c|}
\hline \multirow[t]{2}{*}{ Variables } & \multicolumn{3}{|l|}{ EFS } & \multicolumn{3}{|l|}{ LF } \\
\hline & 5 -year $\mathrm{Cl}$ & HR $(95 \% \mathrm{Cl})$ & $p$ & 5-year Cl & $\mathrm{HR}(95 \% \mathrm{Cl})$ & $p$ \\
\hline All patients & $62.1 \%$ & - & - & $6.9 \%$ & - & - \\
\hline \multicolumn{7}{|l|}{ Age group } \\
\hline$\leq 15$ years & $68.0 \%$ & 1.00 & & $4.6 \%$ & 1.00 & \\
\hline$>15$ years & $47.6 \%$ & $2.00(0.91-4.41)$ & 0.087 & $13.8 \%$ & $3.11(0.44-22.1)$ & 0.257 \\
\hline \multicolumn{7}{|l|}{ Sex } \\
\hline Male & $64.0 \%$ & 1.00 & & $9.1 \%$ & 1.00 & \\
\hline Female & $58.6 \%$ & $1.30(0.60-2.83)$ & 0.513 & $4.8 \%$ & $0.58(0.06-5.53)$ & 0.575 \\
\hline \multicolumn{7}{|l|}{ Risk group } \\
\hline Low & $73.7 \%$ & 1.00 & & $5.6 \%$ & 1.00 & \\
\hline High & $48.2 \%$ & $1.74(0.80-3.80)$ & 0.163 & $8.3 \%$ & $1.33(0.19-9.41)$ & 0.779 \\
\hline \multicolumn{7}{|l|}{ Tumor size } \\
\hline$\leq 8 \mathrm{~cm}$ & $61.1 \%$ & 1.00 & & - & - & - \\
\hline$>8 \mathrm{~cm}$ & $58.1 \%$ & $1.07(0.41-2.76)$ & 0.892 & - & - & - \\
\hline \multicolumn{7}{|l|}{ Necrosis Index } \\
\hline$\leq 95 \%$ & $60.7 \%$ & $1.38(0.54-3.57)$ & 0.503 & $5.6 \%$ & $0.41(0.04-4.52)$ & 0.466 \\
\hline$>95 \%$ & $71.3 \%$ & 1.00 & & $10.5 \%$ & 1.00 & \\
\hline \multicolumn{7}{|l|}{ LDH } \\
\hline$\geq 1.5 \times$ ULN & $51.3 \%$ & $1.11(0.44-2.77)$ & 0.832 & $15.4 \%$ & $6.04(0.55-66.7)$ & 0.142 \\
\hline$<1.5 \times$ ULN & $63.1 \%$ & 1.00 & & $2.4 \%$ & 1.00 & \\
\hline \multicolumn{7}{|l|}{ Pelvic location } \\
\hline Yes & $41.1 \%$ & $1.47(0.55-3.90)$ & 0.440 & - & - & - \\
\hline No & $66.7 \%$ & 1.00 & & - & - & - \\
\hline Radiation dose & - & $0.99(0.98-1.01)$ & 0.560 & - & $0.98(0.86-1.12)$ & 0.796 \\
\hline
\end{tabular}

EFS event-free survival, $L F$ local failure, $C l$ cumulative incidence, $H R$ hazard ratio; $95 \% \mathrm{Cl}, 95 \%$ confidence interval, $L D H$ lactate dehydrogenase, ULN upper limit of normal

Surgery is reserved for situations in which the tumor can be resected with adequate margins, that is, with no evidence of residual disease. Although based on observational studies, local recurrence and survival have shown better results in patients submitted to neoadjuvant chemotherapy and surgery compared to patients submitted to neoadjuvant chemotherapy and radiotherapy [16, $27,28]$. DuBois et al. analyzed using propensity scores the risk of LF and survival in 465 patients with localized ES of bone and found that radiotherapy had a higher risk of local recurrence and death than surgery alone [13]. In the EWING1 trial, radiation therapy showed worse results in terms of EFS $(p=0.009)$ than surgery and PORT. These findings should be analyzed with caution because $70 \%(9 / 13)$ of the patients subjected to radiation had unresectable tumors; 10 patients had tumors located in the spine and pelvis and 3 developed secondary myeloproliferative neoplasms at the beginning of the follow-up period. Due to the small number of local recurrences $(n=4)$, there was no significant difference in LF rates by local control modality.
Several studies included only patients with pelvic ES to investigate possible associations between local control modality and treatment failure [19, 29-31]. Raciborska et al. found that survival was higher in patients treated with surgery and PORT than in those treated with radiotherapy alone ( $81 \%$ and $78 \%$ vs $36 \%$ at 3 years, respectively) [29]. In the present study, 10 patients had pelvic tumors, and $50 \%$ of these patients were treated with definitive radiotherapy $(p=0.012)$. As expected, survival was considerably lower in patients with pelvic compared to non-pelvic tumors $(41.1 \%$ vs $66.7 \%, p=0.44)$. There was no difference in the incidence of LF and survival between the different local control measures in the pelvis.

Nowadays, definitive radiation is an almost exclusive indication for unresectable tumors and for patients with poorer prognosis for whom surgical procedures may be exceptionally mutilating. Advances in radiation technology and multidisciplinary approach have enhanced local control and decreased complications in healthy tissues surrounding tumors. Studies analyzing the use of radiation alone reported 5-year local control rates ranging from 53 to $86 \%$ 


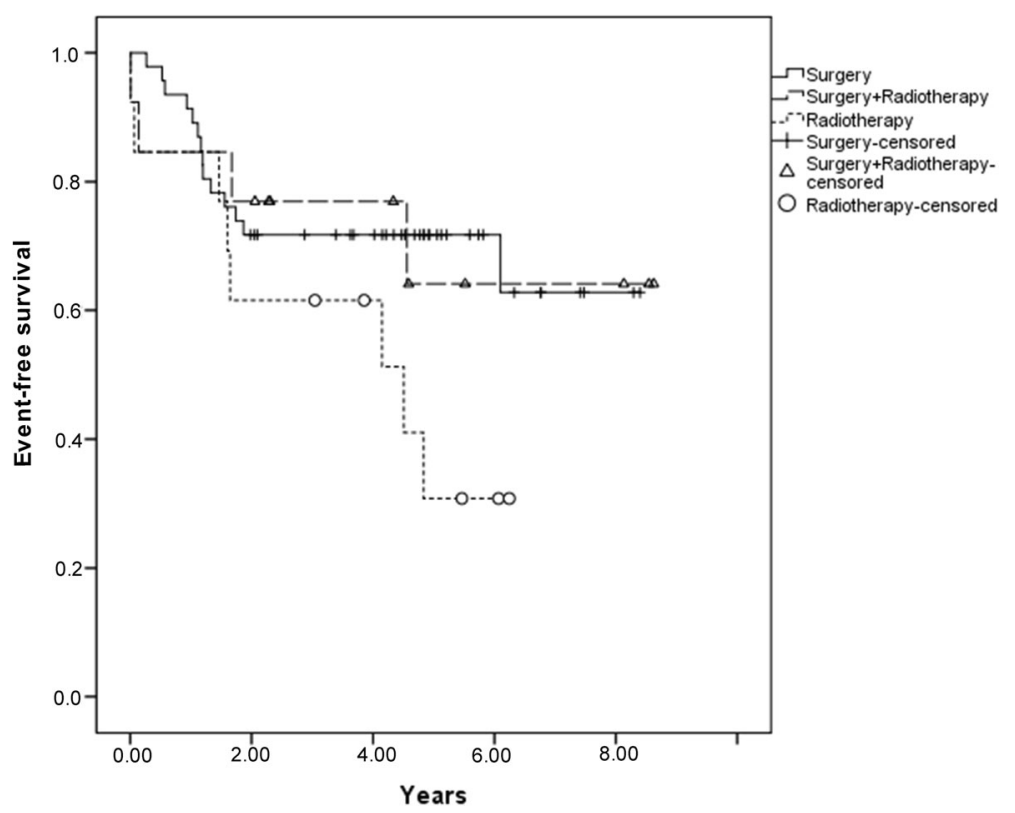

Fig. 1 Event-free survival according to the local treatment modality

with doses between 45 and 65 Gy [9-11, 26, 32, 33]. The EWING1 trial demonstrated that most patients with unresectable tumors and tumors located in the spine and pelvis were treated with definitive radiotherapy. Considerably worse results were obtained in patients treated with radiotherapy alone than in those treated with surgery and PORT. This may be due to high disease morbidity, suboptimal local control with radiotherapy alone, or a combination of these and other factors. The differing clinical characteristics of the radiotherapy group precluded a perfectly reliable comparison between the different local treatment modalities.

Moreover, EWING1's sample was characterized by patients with many risk factors associated with poor prognosis. Forty-eight percent were in the HRG, and more than half had tumors $>8 \mathrm{~cm}$ and were poorer responders

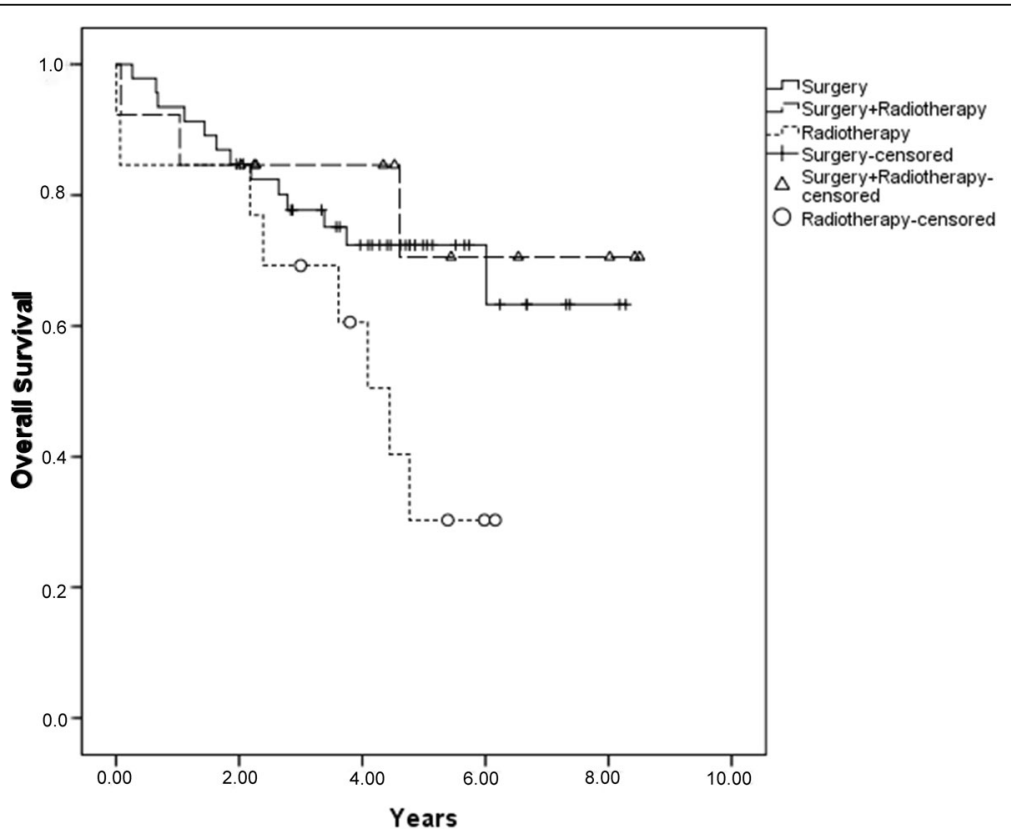

Fig. 2 Overall survival according to the local treatment modality 


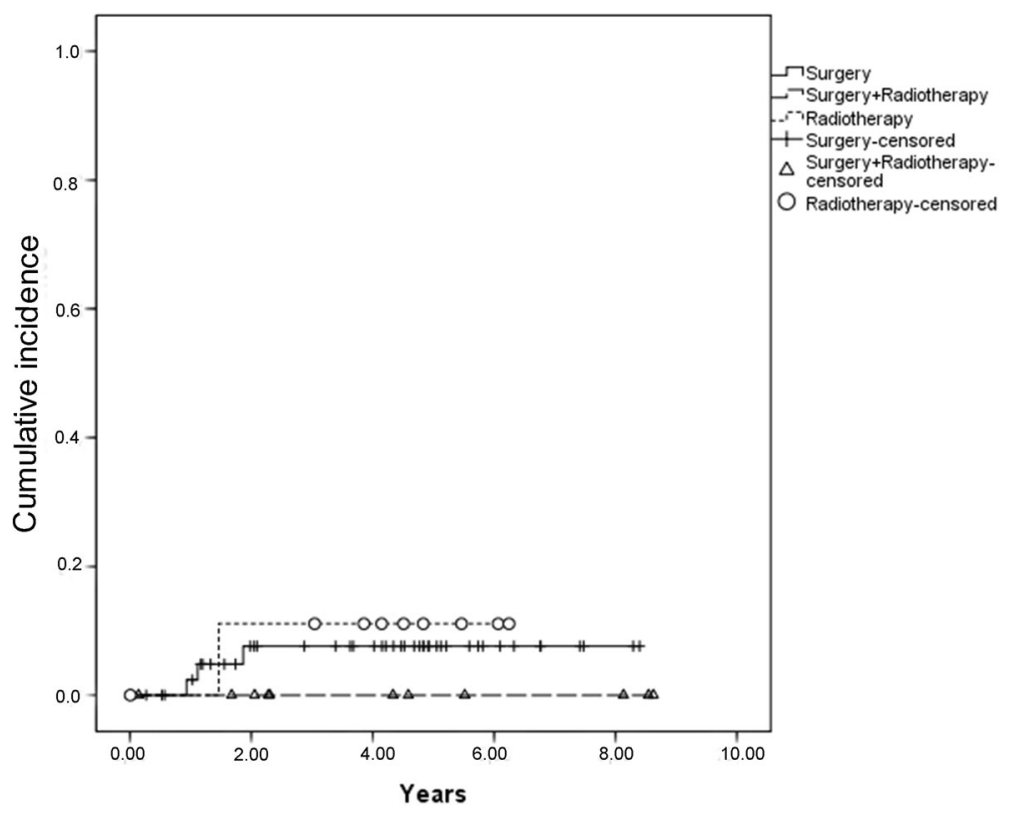

Fig. 3 Cumulative incidence of isolated local recurrence in relation to local treatment modality

to chemotherapy. These worse characteristics suggest a delay in ES diagnosis probably related to social and economic issues from a developing country. Furthermore, higher resistance to chemotherapy could be related to both larger tumors and a specific resistance profile of the patients. Despite all this, for 73 patients included in the current study, the remission rate was $78 \%$.
In summary, we observed similar results to those published by large international cooperative groups $[5,16$, $19,34]$. Every effort made to provide training to local investigators, gather data, and monitor the progress of the first Brazilian protocol for ES has allowed us to describe the different local control strategies used in the treatment of ES in a country of continental size like Brazil. The great

Table 3 Results of multivariate analysis for independent variables associated with death and EFS

\begin{tabular}{|c|c|c|c|c|}
\hline & EFS & $p$ & LF & $p$ \\
\hline Variables & HR (95\% Cl) & & HR (95\% Cl) & \\
\hline \multicolumn{5}{|l|}{ Type of treatment } \\
\hline Surgery & 1.00 & & 1.00 & \\
\hline Surgery + Radiotherapy & $0.88(0.28-2.74)$ & 0.829 & * & * \\
\hline Radiotherapy & $1.84(0.63-5.41)$ & 0.267 & $1.01(0.08-12.8)$ & 0.991 \\
\hline Age group & & & & - \\
\hline$\leq 15$ years & 1.00 & & - & \\
\hline$>15$ years & $2.12(0.96-4.71)$ & 0.064 & - & \\
\hline Risk group & & & & - \\
\hline Low & 1.00 & & - & \\
\hline High & $1.41(0.53-3.71)$ & 0.489 & - & \\
\hline \multicolumn{5}{|l|}{ LDH } \\
\hline$\geq 1.5 \times$ ULN & - & & $6.28(0.50-79.1)$ & 0.155 \\
\hline$<1.5 \times$ ULN & - & & 1.00 & \\
\hline
\end{tabular}

*It is not possible to estimate risk because the interval tends to infinity

EFS event-free survival, $L F$ local failure, $H R$ hazard ratio, $95 \% \mathrm{Cl}, 95 \%$ confidence interval, $L D H$ lactate dehydrogenase, ULN upper limit of normal 
economic, cultural and social diversity of patients as well as the different levels of knowledge of health professionals on the topic make clear the importance of a collaborative approach for a study of this magnitude.

\section{Conclusion}

The EWING1 trial found no significant difference in local or systemic disease recurrence between different treatment modalities. However, regarding survival, there was a significant difference between surgery, radiotherapy, and PORT.

The Brazilian Collaborative Study Group for treatment of ESFT has now been incorporated into the newly formed Latin American Pediatric Oncology Group (GALOP) and a second ESFT study was activated in 2011 [28]. The next step is intended to analyze and report the impact of local control in the second ESFT study.

\begin{abstract}
Abbreviations
95\% Cl: 95\% confidence interval; Cl: Cumulative incidence; CT: Computed tomography; DF: Distant failure; EFS: Event-free survival; ES: Ewing sarcoma; ESFT: Ewing sarcoma family of tumors; HR: Hazard ratio; HRG: High-risk group; ICE: Ifosfamide, carboplatin, and etoposide; IE: Ifosfamide and etoposide; LDH: Lactate dehydrogenase; LF: Local failure; LRG: Low-risk group; MRI: Magnetic resonance imaging; OS: Overall survival; ULN: Upper limit of normal; VDC: Vincristine, doxorubicin, and cyclophosphamide
\end{abstract}

\section{Acknowledgements}

Not applicable

\section{Funding}

This work was financially supported by the Children's Cancer Institute and Rafael Accordi Foundation, Porto Alegre, RS, Brazil. The funders had no role in study design, data collection and analysis, decision to publish, or preparation of the manuscript.

\section{Availability of data and materials}

The datasets used and/or analyzed during the current study are available from the corresponding author on reasonable request.

\section{Authors' contributions}

$R G B, L J G, R J F$, and $A L B$ contributed to the analysis and interpretation of the patient data regarding local control modalities and were involved in drafting the manuscript. CRG and RGB are the heads of the Department of Orthopedic Research and revised the manuscript critically for important intellectual content. JFCS contributed to the acquisition, analysis and interpretation of data. RJF, EAT, GB, SAN, AD, AMB, ESY, OAS, VP, RGB, and LJG contributed to the conception and design of the study and included more than 5 patients from their institutions. All authors read and approved the final manuscript.

\section{Competing interests}

The authors declare that they have no competing interests.

\section{Consent for publication \\ Not applicable}

\section{Ethics approval and consent to participate}

The study was approved by the institutional review board of Hospital de Clínicas de Porto Alegre through the Office of Research and Graduate Studies (IRB No. 00000921). All patients signed an informed consent form prior to their inclusion in the EWING1 trial from 2003 to 2010.

\section{Publisher's Note}

Springer Nature remains neutral with regard to jurisdictional claims in published maps and institutional affiliations.

\section{Author details}

${ }^{1}$ Service of Orthopedics and Traumatology, Hospital de Clínicas de Porto Alegre (HCPA), Rua Ramiro Barcelos, 2350, Bairro Santa Cecilia, Porto Alegre, RS, 90035-903, Brazil. ${ }^{2}$ Department of Pediatrics, HCPA, Universidade Federal do Rio Grande do Sul (UFRGS), Porto Alegre, Brazil. ${ }^{3}$ Department of Pediatrics, Hospital São Lucas, Pontifícia Universidade Católica do Rio Grande do Sul (PUCRS), Porto Alegre, RS, Brazil. ${ }^{4}$ Service of Orthopedics and Traumatology, HCPA, Porto Alegre, RS, Brazil. ${ }^{5}$ Support Group for Children and Adolescents with Cancer (GRAACC), Universidade Federal de São Paulo (UNIFESP), São Paulo, SP, Brazil. ${ }^{6}$ Fundação Pio XII, Hospital de Câncer Infantojuvenil, Barretos, SP, Brazil. ' Centro Hospitalario Pereira Rossell, Montevideo, Uruguay. ${ }^{8}$ Orthopedics Service, Hospital A.C. Camargo Cancer Center, São Paulo, SP, Brazil. ' ${ }^{9}$ Service of Orthopedics and Traumatology, Santa Casa de Misericórdia de Porto Alegre, Porto Alegre, RS, Brazil.

${ }^{10}$ Orthopedic Trauma Institute, Hospital das Clínicas de São Paulo, School of Medicine, Universidade de São Paulo (USP), São Paulo, SP, Brazil.

${ }^{11}$ Department of Orthopedics and Traumatology, Santa Casa de Misericórdia de São Paulo (HSCSP), São Paulo, SP, Brazil. ${ }^{12}$ Service of Orthopedics and Traumatology, Hospital São Lucas, Pontifícia Universidade Católica do Rio Grande do Sul (PUCRS), Porto Alegre, RS, Brazil. ${ }^{13}$ Hospital das Clínicas de Botucatu, School of Medicine, Universidade Estadual Paulista (UNESP), Botucatu, SP, Brazil. ${ }^{14}$ Instituto do Câncer Infantil, Porto Alegre, RS, Brazil. ${ }^{15}$ Instituto do Câncer Infantil, Porto Alegre, RS, Brazil.

Received: 6 February 2017 Accepted: 26 May 2017

Published online: 15 June 2017

\section{References}

1. Grier HE. The Ewing family of tumors. Ewing's sarcoma and primitive neuroectodermal tumors. Pediatr Clin N am. 1997;44:991-1004.

2. Ferrari S, Sundby Hall K, Luksch R, Tienghi A, Wiebe T, Fagioli F, et al. Nonmetastatic Ewing family tumors: high-dose chemotherapy with stem cell rescue in poor responder patients. Results of the Italian sarcoma group/ Scandinavian sarcoma group III protocol. Ann Oncol. 2011;22:1221-7.

3. Gupta AA, Pappo A, Saunders N, Hopyan S, Ferguson P, Wunder J, et al. Clinical outcome of children and adults with localized Ewing sarcoma: impact of chemotherapy dose and timing of local therapy. Cancer. 2010; 116:3189-94.

4. Gaspar N, Hawkins DS, Dirksen U, Lewis IJ, Ferrari S, Le Deley MC, et al. Ewing sarcoma: current management and future approaches through collaboration. J Clin Oncol. 2015;33:3036-46.

5. Grier HE, Krailo MD, Tarbell NJ, Link MP, Fryer CJ, Pritchard DJ, et al. Addition of ifosfamide and etoposide to standard chemotherapy for Ewing's sarcoma and primitive neuroectodermal tumor of bone. N Engl J med. 2003;348:694-701.

6. Paulussen M, Craft AW, Lewis I, Hackshaw A, Douglas C, Dunst J, et al. Results of the EICESS-92 study: two randomized trials of Ewing's sarcoma treatment-cyclophosphamide compared with ifosfamide in standard-risk patients and assessment of benefit of etoposide added to standard treatment in high-risk patients. J Clin Oncol. 2008;26:4385-93.

7. Oberlin O, Rey A, Desfachelles AS, Philip T, Plantaz D, Schmitt C, et al. Impact of high-dose busulfan plus melphalan as consolidation in metastatic Ewing tumors: a study by the Societe Francaise des cancers de l'Enfant. J Clin Oncol. 2006;24:3997-4002.

8. Delattre O, Zucman J, Melot T, Garau XS, Zucker JM, Lenoir GM, et al. The Ewing family of tumors-a subgroup of small-round-cell tumors defined by specific chimeric transcripts. N Engl J med. 1994;331:294-9.

9. Burgert EO Jr, Nesbit ME, Garnsey LA, Gehan EA, Herrmann J, Vietti TJ, et al. Multimodal therapy for the management of nonpelvic, localized Ewing's sarcoma of bone: intergroup study IESS-II. J Clin Oncol. 1990;8:1514-24.

10. Nesbit ME Jr, Gehan EA, Burgert EO Jr, Vietti TJ, Cangir A, Tefft M, et al. Multimodal therapy for the management of primary, nonmetastatic Ewing's sarcoma of bone: a long-term follow-up of the first intergroup study. J Clin Oncol. 1990;8:1664-74.

11. Craft A, Cotterill S, Malcolm A, Spooner D, Grimer R, Souhami R, et al. Ifosfamide-containing chemotherapy in Ewing's sarcoma: the second United Kingdom Children's Cancer study group and the Medical Research Council Ewing's tumor study. J Clin Oncol. 1998;16:3628-33. 
12. Schuck A, Ahrens S, Paulussen M, Kuhlen M, Konemann S, Rube C, et al. Local therapy in localized Ewing tumors: results of 1058 patients treated in the CESS 81, CESS 86, and EICESS 92 trials. Int J Radiat Oncol Biol Phys. 2003;55:168-77.

13. DuBois SG, Krailo MD, Gebhardt MC, Donaldson SS, Marcus KJ, Dormans J, et al. Comparative evaluation of local control strategies in localized Ewing sarcoma of bone: a report from the Children's oncology group. Cancer. 2015;121:467-75.

14. Foulon S, Brennan B, Gaspar N, Dirksen U, Jeys L, Cassoni A, et al. Can postoperative radiotherapy be omitted in localised standard-risk Ewing sarcoma? An observational study of the euro-E.W.I.N.G group. Eur J Cancer. 2016;61:128-36.

15. Bacci G, Forni C, Longhi A, Ferrari S, Donati D, De Paolis M, et al. Long-term outcome for patients with non-metastatic Ewing's sarcoma treated with adjuvant and neoadjuvant chemotherapies. 402 patients treated at Rizzoli between 1972 and 1992. Eur J Cancer. 2004;40:73-83.

16. Shankar AG, Pinkerton CR, Atra A, Ashley S, Lewis I, Spooner D, et al. Local therapy and other factors influencing site of relapse in patients with localised Ewing's sarcoma. United Kingdom Children's Cancer study group (UKCCSG). Eur J Cancer. 1999;35:1698-704.

17. Donaldson SS. Ewing sarcoma: radiation dose and target volume. Pediatr Blood Cancer. 2004;42:471-6.

18. Hosalkar HS, Dormans JP. Limb sparing surgery for pediatric musculoskeletal tumors. Pediatr Blood Cancer. 2004;42:295-310.

19. Yock TI, Krailo M, Fryer CJ, Donaldson SS, Miser JS, Chen Z, et al. Local control in pelvic Ewing sarcoma: analysis from INT-0091-a report from the Children's oncology group. J Clin Oncol. 2006;24:3838-43.

20. Brunetto AL, Castillo LA, Petrilli AS, Macedo CD, Boldrini E, Costa C, et al. Carboplatin in the treatment of Ewing sarcoma: results of the first Brazilian collaborative study group for Ewing sarcoma family tumors-EWING1. Pediatr Blood Cancer. 2015;62:1747-53

21. Krasin MJ, Rodriguez-Galindo C, Davidoff AM, Billups CA, Fuller CE, Neel MD, et al. Efficacy of combined surgery and irradiation for localized Ewing's sarcoma family of tumors. Pediatr Blood Cancer. 2004;43:229-36.

22. Picci $P$, Bohling $T$, Bacci G, Ferrari S, Sangiorgi L, Mercuri $M$, et al. Chemotherapy-induced tumor necrosis as a prognostic factor in localized Ewing's sarcoma of the extremities. J Clin Oncol. 1997;15:1553-9.

23. Nesbit ME Jr, Perez CA, Tefft M, Burgert EO Jr, Vietti TJ, Kissane J, et al. Multimodal therapy for the management of primary, nonmetastatic Ewing's sarcoma of bone: an intergroup study. Natl Cancer Inst Monogr. 1981:255-62.

24. Zahlten-Hinguranage A, Bernd L, Ewerbeck V, Sabo D. Equal quality of life after limb-sparing or ablative surgery for lower extremity sarcomas. Br J Cancer. 2004;91:1012-4.

25. Germain MA, Mascard E, Dubousset J, Nguefack M. Free vascularized fibula and reconstruction of long bones in the child-our evolution. Microsurgery. 2007;27:415-9.

26. Donaldson SS, Torrey M, Link MP, Glicksman A, Gilula L, Laurie F, et al. A multidisciplinary study investigating radiotherapy in Ewing's sarcoma: end results of POG \#8346. Pediatric oncology group. Int J Radiat Oncol Biol Phys. 1998:42:125-35.

27. Womer RB, West DC, Krailo MD, Dickman PS, Pawel BR, Grier HE, et al. Randomized controlled trial of interval-compressed chemotherapy for the treatment of localized Ewing sarcoma: a report from the Children's oncology group. J Clin Oncol. 2012;30:4148-54.

28. Bacci G, Ferrari S, Bertoni F, Rimondini S, Longhi A, Bacchini P, et al. Prognostic factors in nonmetastatic Ewing's sarcoma of bone treated with adjuvant chemotherapy: analysis of 359 patients at the Istituto Ortopedico Rizzoli. J Clin Oncol. 2000;18:4-11.

29. Raciborska A, Bilska K, Rychlowska-Pruszynska M, Drabko K, Chaber R, Pogorzala $M$, et al. Internal hemipelvectomy in the management of pelvic Ewing sarcoma - are outcomes better than with radiation therapy? J Pediatr Surg. 2014;49:1500-4.

30. Scully SP, Temple HT, O'Keefe RJ, Scarborough MT, Mankin HJ, Gebhardt MC. Role of surgical resection in pelvic Ewing's sarcoma. J Clin Oncol. 1995; 13:2336-41.

31. Carrie C, Mascard E, Gomez F, Habrand JL, Alapetite C, Oberlin O, et al. Nonmetastatic pelvic Ewing sarcoma: report of the French society of pediatric oncology. Med Pediatr Oncol. 1999;33:444-9.

32. Ahrens S, Hoffmann C, Jabar S, Braun-Munzinger G, Paulussen M, Dunst J, et al. Evaluation of prognostic factors in a tumor volume-adapted treatment strategy for localized Ewing sarcoma of bone: the CESS 86 experience. Cooperative Ewing sarcoma study. Med Pediatr Oncol. 1999;32:186-95.
33. Dunst J, Jurgens $H$, Sauer $R$, Pape $H$, Paulussen M, Winkelmann W, et al. Radiation therapy in Ewing's sarcoma: an update of the CESS 86 trial. Int Radiat Oncol Biol Phys. 1995;32:919-30.

34. Dunst J, Sauer R, Burgers JM, Hawliczek R, Kurten R, Winkelmann W, et al. Radiation therapy as local treatment in Ewing's sarcoma. Results of the cooperative Ewing's sarcoma studies CESS 81 and CESS 86. Cancer. 1991;67:2818-25.

\section{Submit your next manuscript to BioMed Central and we will help you at every step:}

- We accept pre-submission inquiries

- Our selector tool helps you to find the most relevant journal

- We provide round the clock customer support

- Convenient online submission

- Thorough peer review

- Inclusion in PubMed and all major indexing services

- Maximum visibility for your research

Submit your manuscript at www.biomedcentral.com/submit 